\title{
Transient hyperglycaemia - an underestimated problem of paediatric oncohaematology
}

\author{
Ninela Irga ${ }^{1}$, Malgorzata Mysliwiec ${ }^{1}$, Marcelina Osak ${ }^{1}$, Malgorzata Szmigiero-Kawko ${ }^{1}$, \\ Elzbieta Adamkiewicz-Drozynska', Radoslaw Jaworski
}

\author{
1Department of Paediatrics, Haematology, Oncology and Endocrinology, Medical \\ University of Gdansk, Poland \\ 2Department of Paediatric Cardiac Surgery, Mikolaj Kopernik Pomeranian Centre \\ of Traumatology, Gdansk, Poland
}

Submitted: 17 October 2011

Accepted: 4 February 2012

Arch Med Sci 2012; 8, 4: 672-677

DOI: 10.5114/aoms.2012.30291

Copyright @ 2012 Termedia \& Banach

\section{Abstract:}

Introduction: The majority of hyperglycaemic incidents in oncohaematological patients treated with glucocorticosteroids remain undiagnosed. The aim of our study was to work out a detailed protocol for the control of carbohydrate metabolism and to evaluate whether such a protocol can help in diagnosis of carbohydrate metabolism disturbances in oncohaematological paediatric patients.

Material and methods: A one hundred and twenty-eight children treated for proliferative diseases of the haematopoietic system and severe aplastic anaemia with therapeutic protocols including glucocorticosteroids were divided into two groups. Group I consisted of 70 children, whose blood glucose was evaluated on random occasions (retrospective analysis). Group II consisted of 58 children included in the programme of intensive carbohydrate metabolism control (prospective analysis). We compared the incidence of hyperglycaemia in both groups as well as the number of hyperglycaemic incidents per individual therapeutic protocol.

Results: A significantly higher incidence of transient hyperglycaemia was noted in oncohaematological patients in the programme of early carbohydrate metabolism disturbances diagnosis than in the other group (22.4\% vs. $5.7 \%$ respectively; $p=0.008$ ), especially in patients treated with the ALL IC-BFM 2002 protocol for the high risk group (arm A and B), the ALL-REZ BFM 2002 protocol, and in a heterogenous group of children (protocols ALCL 99, Euro-LB02, Interfant-06, WPSAA) ( $p=0.042,0.021$ and 0.002 , respectively).

Conclusions: The improvement of transient hyperglycaemia detection may constitute the first step towards the reduction of unfavourable consequences of hyperglycaemia. Prospective studies are required to demonstrate the influence of normal carbohydrate metabolism on the frequency of infectious complications in this group.

Key words: glucocorticosteroids, oncohaematological children, prospective study, transient hyperglycaemia.

\section{Introduction}

Transient hyperglycaemia ( $\mathrm{TH}$ ) may occur during the treatment of proliferative diseases of the haematopoietic system and severe aplastic anaemia. The TH is usually connected with administration of glucocorticosteroids, but other diabetogenic factors, such as L-asparaginase, are also used in the

\author{
Corresponding author: \\ Ninela Irga MD, PhD \\ Department of Paediatrics, \\ Haematology, \\ Oncology and Endocrinology \\ Medical University of Gdansk \\ 7 Debinki \\ 80-210 Gdansk, Poland \\ Phone/fax: +48583492863 \\ E-mail: nirga@gumed.edu.pl
}


treatment of acute lymphoblastic leukaemia [1-3]. The incidence of hyperglycaemia in the course of steroid administration ranges according to various authors from $16 \%$ to $35 \%$ in paediatric haematological diseases $[4,5]$. The negative influence of hyperglycaemia on the human immune system is well acknowledged $[6,7]$. In children undergoing immunosuppressive treatment hyperglycaemia may represent a significant risk factor for a life-threatening infection. Considering the high incidence of hyperglycaemia and threats it poses for the haematological patients, early diagnosis and the introduction of adequate treatment should be prioritised. However, TH-related ketoacidosis is infrequent; therefore diagnosis based on clinical symptoms is difficult or simply impossible [1]. Only an active search for hyperglycaemia in patients receiving glucocorticosteroid therapy enables diagnosis and introduction of appropriate treatment allowing for reduction of hyperglycaemia negative effects. We postulate that the majority of hyperglycaemic incidents in oncohaematological patients treated with glucocorticosteroids remain undiagnosed. The improvement of $\mathrm{TH}$ detection may constitute the first step towards the reduction of unfavourable consequences of hyperglycaemia in this group [8]. The aim of this study was to work out a detailed protocol for carbohydrate metabolism control and to evaluate whether the protocol influences the frequency of diagnosed incidents of carbohydrate metabolism disturbances in children treated for acute lymphoblastic leukaemia (ALL), nonHodgkin lymphoma (NHL) and severe aplastic anaemia (SAA).

\section{Material and methods}

The study group included 128 children treated due to proliferative diseases of the haematopoietic system and severe aplastic anaemia in the Department of Paediatrics, Haematology, Oncology and Endocrinology at the Medical University of Gdansk from October 2005 to December 2010. The only inclusion cri- terion was the use of glucocorticosteroids in the therapeutic protocol. The detailed data on drugs used in individual therapeutic protocols, total doses per $\mathrm{m}^{2}$ of body surface and numbers of chemotherapy cycles with steroids in each protocol are presented in Table I. All patients were divided into two groups. Group I consisted of 70 children treated from October 2005 to October 2008, in whom random blood glucose was evaluated as the only parameter (retrospective analysis). Group II consisted of 58 children treated from October 2008 to December 2010, in whom a programme of intensive carbohydrate metabolism control was implemented (prospective analysis). In all the patients in group II, the following data were assessed prior to steroid administration: family history of diabetes mellitus, body mass index (BMI) and levels of insulin, C-peptide, and glycosylated haemoglobin $\left(\mathrm{HbA}_{1 \mathrm{c}}\right)$. The glucose tolerance test was not performed due to patients' general condition caused by their primary disease. All patients in group II had the fasting blood glucose checked daily during the glucocorticosteroid administration period, and in the subgroup of higher risk when fasting blood glucose was abnormal - blood glucose was regularly evaluated after meals. The subgroup of higher risk consisted of patients who fulfilled at least one of the following criteria: C-peptide $<0.9$ or $>2.5 \mathrm{ng} / \mathrm{ml}$, fasting insulin $<3$ or $>15 \mathrm{mU} /$, family history of diabetes mellitus or obesity in first- or second-degree relatives and $\mathrm{BMI}>$ the $95^{\text {th }}$ percentile. Whenever any disturbances in carbohydrate metabolism were noted, infusion fluids with glucose were discontinued, a reduced carbohydrate diet was introduced and blood glucose was monitored systematically (before breakfast and $2 \mathrm{~h}$ after main meals). When diabetes mellitus was diagnosed, insulin was started (basic or intensive insulin therapy). Three therapeutic management protocols were developed for children on glucocorticosteroids, named A, B, and C. Protocol A was applied in the cases where glucocorticosteroids were introduced for the first time or there were no carbohydrate

Table I. Chemotherapy protocols used in the analysed patients in regard to total steroid dose, drug type and number of cycles with steroids

\begin{tabular}{|llc|}
\hline Chemotherapy protocol & $\begin{array}{l}\text { Total dose of steroids in chemotherapy } \\
\text { protocol }\left[\mathrm{mg} / \mathrm{m}^{2}\right] \text { and type of drug }\end{array}$ & $\begin{array}{c}\text { Number of } \\
\text { chemotherapy } \\
\text { cycles with steroids }\end{array}$ \\
\hline $\begin{array}{l}\text { ALL IC-BFM } 2002 \text { standard-risk } \\
\text { group arm and intermediate-risk } \\
\text { group arm }\end{array}$ & $\begin{array}{l}\text { Prednisone } 1740 \mathrm{mg} / \mathrm{m}^{2} \text {; Dexamethasone } \\
236.25 \mathrm{mg} / \mathrm{m}^{2}\end{array}$ & 2 \\
\hline $\begin{array}{l}\text { ALL IC-BFM } 2002 \text { high-risk group } \\
\text { arm A and B }\end{array}$ & $\begin{array}{l}\text { Prednisone } 1740 \mathrm{mg} / \mathrm{m}^{2} \text {; Dexamethasone } \\
600 \mathrm{mg} / \mathrm{m}^{2}\end{array}$ & 7 \\
\hline ALL-REZ BFM 2002 & Dexamethasone $500-700 \mathrm{mg} / \mathrm{m}^{2}$ & $5-7$ \\
\hline B-NHL BFM 04 (arm: 2, 3 and 4) & Dexamethasone $100-490 \mathrm{mg} / \mathrm{m}^{2}$ & $2-7$ \\
\hline Other* & Prednisone $210-1740 \mathrm{mg} / \mathrm{m}^{2} ;$ Dexamethasone $236.25-500 \mathrm{mg} / \mathrm{m}^{2}$ & $2-6$ \\
\hline
\end{tabular}

${ }^{*}$ ALCL 99, Euro-LB02, Interfant-06, WPSAA 


\section{Protocol A}

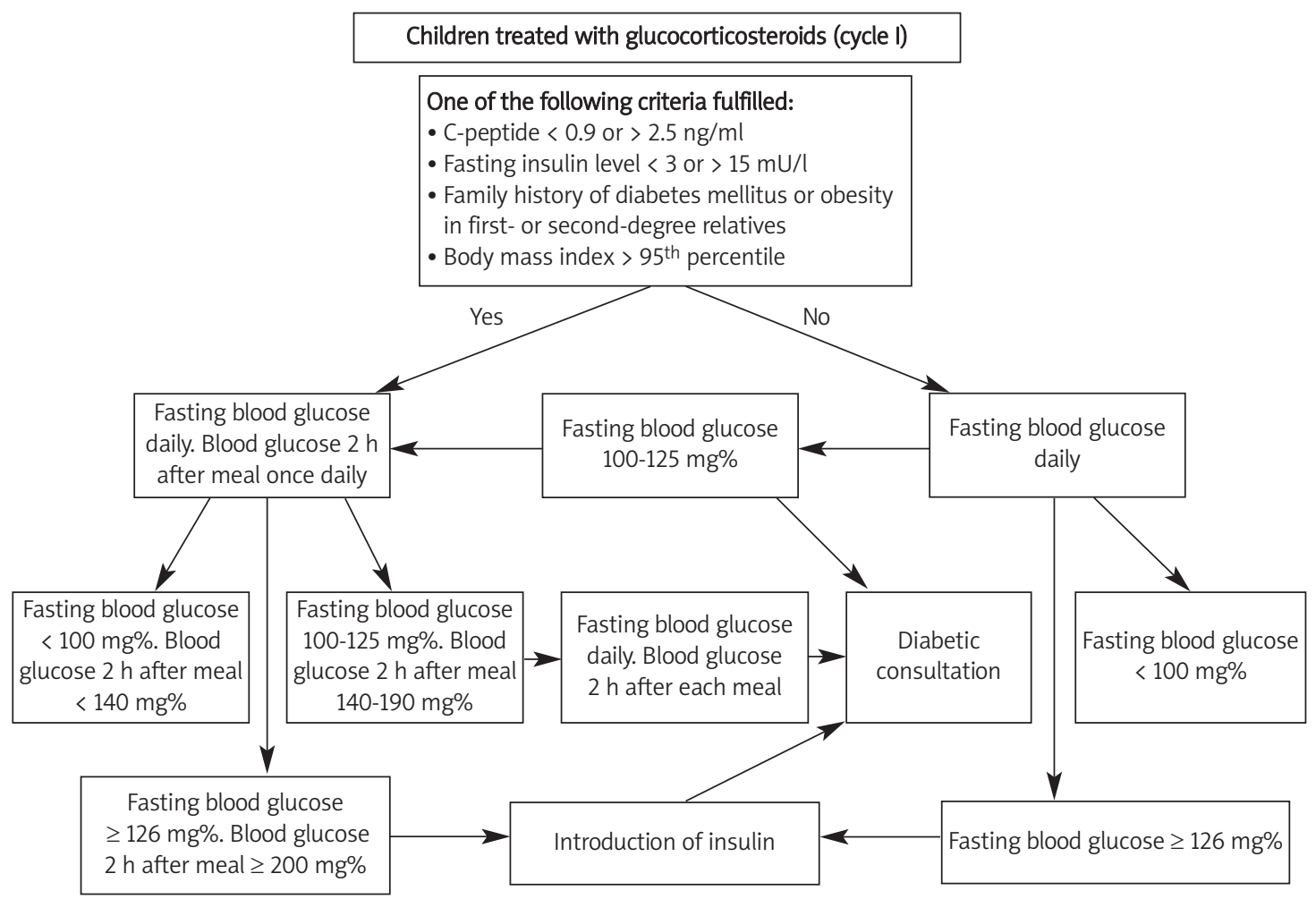

Figure 1. Protocol A for paediatric patients treated for proliferative diseases of the haematopoietic system and severe aplastic anaemia after first introduction of glucocorticosteroids or without carbohydrate metabolism disorders in the previous treatment cycle

Protocol B

\section{Children treated with glucocorticosteroids (cycle II)}

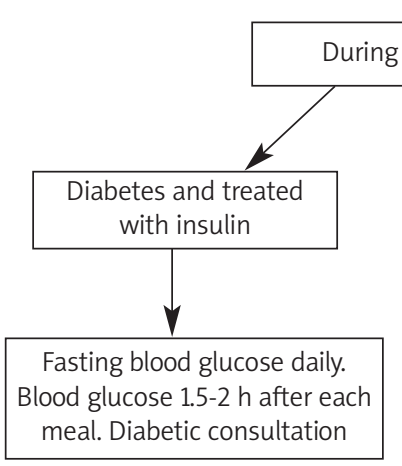

Protocol C

\section{Children treated with glucocorticosteroids (cycle III and consecutive)}

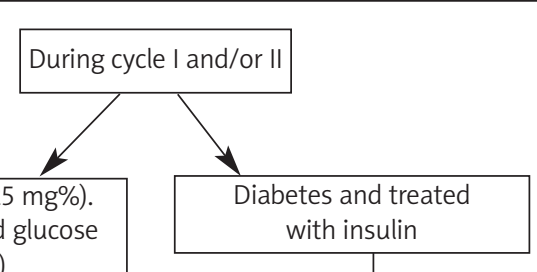

Abnormal carbohydrate tolerance (blood glucose 1.5-2 $\mathrm{h}$ after meal $140-199 \mathrm{mg} \%$ )

Fasting blood glucose daily. Blood glucose 1.5-2 $\mathrm{h}$ after meal once daily. Reduced carbohydrate diet and management as for cycle I

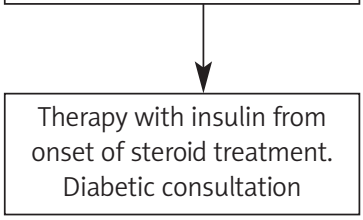

Figure 2. Protocol B and C for paediatric patients treated for proliferative diseases of the haematopoietic system and severe aplastic anaemia. Protocol B was applied for children with carbohydrate metabolism disorders in the first cycle; protocol C was applied for children with carbohydrate metabolism disorders in two subsequent cycles

metabolism disturbances (CHMD) observed in the previous cycle (Figure 1). If there had been CHMD in the first cycle, protocol B was applied in the next one. In the cases of CHMD in any of the two subsequent glucocorticosteroid cycles, protocol C was used (Figure 2).

Group I and II were compared in regard to age, sex, BMI, primary diagnosis, and applied therapeu- 
tic protocols. The total number of therapeutic cycles with glucocorticosteroids was calculated for both groups. The incidence of hyperglycaemia in both groups was comparatively analyzed. Next, the number of hyperglycaemic incidents per individual therapeutic protocol was analyzed in regard to the number of cycles with glucocorticosteroids. The fact that some children in group II were also given L-asparaginase according to the therapeutic protocol was also taken into consideration; we compared the incidence of hyperglycaemia in those who received the drug to those who did not.

The research was approved by the Independent Bioethics Committee for Scientific Research of the Medical University of Gdansk.

\section{Statistical analysis}

All the patients' data and results were encoded and analysed using Microsoft Excel 2007 (Microsoft Inc, USA). The data showing normal distribution were compared using Student's t-test. Other data were analyzed with non-parametric tests (MannWhitney U test). Categorical values were compared using Pearson's $\chi^{2}$ test with the Yates correction or Fisher's exact test when applicable. Statistical analysis was carried out with SPSS v. 13.0 (SPSS Inc., USA) statistical software.

\section{Results}

The age, sex distribution, BMI percentile, primary diagnosis and therapeutic protocols did not differ between the groups (Table II). Four out of 70 patients from group I (5.7\%) (before introducing the programme of CHMD control) had hyperglycaemia diagnosed during the treatment of the primary disease with glucocorticosteroid including protocols. In group II, for which CHMD control was applied, hyperglycaemia was diagnosed in $13 / 58$ patients (22.4\%). The differences in the incidence of hyperglycaemia between the two studied groups was statistically significant $(p=0.008)$. A total of 209 therapeutic cycles with administration of glucocorticosteroids were carried out in group I, with on average 3 cycles (SD 1.8; range 1-7) per patient, and 165 therapeutic cycles were carried out in group II, with on average 2.9 therapeutic cycles (SD 2.1; range 1-8) per patient ( $p=0.209)$. In group I 5 hyperglycaemic incidents were diagnosed in the course of 209 cycles including glucocorticosteroids (2.4\%), whereas in group II 30 hyperglycaemic incidents were diagnosed in over 165 cycles (18.2\%). The detection of hyperglycaemia in group II was significantly higher $(p<0.001)$. The incidence of hyperglycaemia in regard to the primary disease therapeutic protocol is presented in Table III. Higher incidence of hyperglycaemia was observed in group II, i.e. in children included in the programme of CHMD early detection. Statistical significance was observed for patients who were treated with the ALL IC-BFM 2002 protocol for the highrisk group (arm A and B), the treatment protocol for recurrent acute lymphoblastic leukaemia (ALL-REZ BFM 2002), and in a heterogeneous group of children in whom protocols ALCL 99, Euro-LB02, Inter-

Table II. Comparison of the studied groups in regard to age, sex, diagnoses and applied chemotherapy protocols

\begin{tabular}{|lccc|}
\hline Feature & Group I & Group II & Value of $p$ \\
\hline Age (median (SD)) & $6.24(4.38)$ & $5.42(4.46)$ & 0.705 \\
\hline BMI percentile (mean (SD)) & $41.7(34.7)$ & $33.5(35)$ & 0.772 \\
\hline Gender: & & & 0.183 \\
- Male & 34 & 35 & \\
- Female & 36 & 23 & \\
\hline Diagnosis: & & & 0.751 \\
- ALL & 52 & 42 & \\
- ALL recidiva & 4 & 6 & \\
- ALCL & 3 & 0 & \\
- NHL B cell & 7 & 6 & \\
- NHL T cell & 1 & 1 & \\
- NHL recidiva & 1 & 1 & \\
- SAA & 2 & 2 & \\
\hline Chemotherapy protocol: & & & \\
- ALL IC-BFM 2002 standard-risk group arm and intermediate-risk group arm & 41 & 35 & \\
- ALL IC-BFM 2002 high-risk group arm A and B & 9 & 5 & \\
- ALL-REZ BFM 2002 & & & \\
- B-NHL BFM 04 (arm: 2, 3 and 4) & & & \\
- Other* & 4 & 6 & \\
\hline
\end{tabular}

${ }^{*} A L C L$ 99, Euro-LB02, Interfant-06, WPSAA; SD - standard deviation, BMI - body mass index, ALL - acute lymphoblastic leukaemia, $A L C L$ - anaplastic large cell lymphoma, NHL - non-Hodgkin lymphoma, SAA - severe aplastic anaemia 
Table III. Comparison of the studied groups in regard to the incidence of hyperglycaemia depending on the applied therapeutic protocol

\begin{tabular}{|lcccc|}
\hline Chemotherapy protocol & $\begin{array}{c}\text { Incidence of } \\
\text { hyperglycaemia }\end{array}$ & Group I & Group II & Value of $p$ \\
\hline ALL IC-BFM 2002 standard-risk group arm & Yes & 1 & 2 & 0.582 \\
\cline { 2 - 5 } and intermediate-risk group arm & No & 77 & 59 & \\
\hline ALL IC-BFM 2002 high-risk group arm A and B & Yes & 3 & 7 & 0.042 \\
\cline { 2 - 5 } & No & 47 & 25 & 0.021 \\
\hline ALL-REZ BFM 2002 & Yes & 1 & 13 & \\
\cline { 2 - 5 } & No & 19 & 25 & 0.106 \\
\hline B-NHL BFM 04 (arm: 2, 3 and 4) & Yes & 0 & 2 & 14 \\
\hline Other* & No & 32 & 6 & 0.002 \\
\cline { 2 - 5 } & Yes & 0 & 12 & \\
\hline
\end{tabular}

${ }^{*} A L C L$ 99, Euro-LB02, Interfant-06, WPSAA

fant-06 and WPSAA were used ( $p$ was $0.042,0.021$ and 0.002 respectively). Moreover, the incidence of hyperglycaemia did not differ significantly between the group of children treated with protocols including L-asparaginase and the group of children not receiving this drug (19\% vs. $50 \% ; p=0.119)$.

\section{Discussion}

Numerous reports confirm the unfavourable impact of hyperglycaemia on the course of therapy in patients with life-threatening conditions $[8,9]$. In parallel they indicate that better therapeutic effects can be achieved owing to meticulous blood glucose control [2, 3, 8-13]. Many authors emphasize the unfavourable influence of hyperglycaemia on the immune system in humans $[6,14]$. Children treated for proliferative diseases of the haematopoietic system and severe aplastic anaemia are especially exposed to life-threatening infections. New predictors of the final prognosis in these patients are still being sought [15]. Neutropenia, as is well established, is the most significant risk factor for infection in this group [16]. Hyperglycaemia could however be an accumulative and underestimated infection risk factor, especially in children. Therapeutic protocols applied in paediatric oncohaematology lack detailed guidelines regarding CHMD monitoring. Since the typical symptoms of ketoacidosis are absent, many episodes of hyperglycaemia in children treated with glucocorticosteroids remain unnoticed and undiagnosed $[1,17]$. Literature data on hyperglycaemia frequency in this group of patients vary; frequency of $4 \%$ to $22.2 \%$ was reported $[1,3,13,16,17]$. Most of the cited studies were based on retrospective analysis. The data presented in this paper come from a prospectively designed study. Our results, showing significantly higher incidence of diabetes in children included in the programme for early CHMD diagnosis, seem to confirm the hypothesis. It also seems important to preselect a group of patients with a high risk of developing TH within the children treated for proliferative diseases of the haematopoietic system and severe aplastic anaemia.

The algorithm of diagnostic and therapeutic management formulated in this study includes the evaluation of early risk of diabetes development as well as previous incidents of diabetes in preceding chemotherapy cycles. The simple criteria applied, including medical history, BMI evaluation and preliminary assessment of C-peptide level, glucose concentration and glycosylated haemoglobin level, enable planning CHMD control in individual patients. The algorithm allows for early diagnosis and implementation of appropriate therapeutic measures. It may go a long way towards reducing the number of negative effects of hyperglycaemia in the analysed group. The algorithm is simple and uncomplicated, and requires no specialist equipment or financial expenditures.

In conclusion, the protocols of early detection of carbohydrate metabolism disturbances formulated here may be useful in all children on chronic treatment with glucocorticosteroids, not only in those with haematopoietic system diseases, as it seems that also in these children some hyperglycaemia episodes remain undiagnosed. The analysis of how $\mathrm{L}$-asparaginase impacts the incidence of hyperglycaemia showed no statistically significant differences when groups of children receiving or not receiving the drug were compared. However, prospective studies including larger groups are required. Another advantage of the protocol applied here is the opportunity to select a group of children who may develop diabetes in the future. These children must be followed up in the Diabetes Outpa- 
tient Clinic after termination of their antineoplastic therapy. The next expected effect of the protocol formulated here is the reduction of the number of infections in children treated for diseases of the haematopoietic system. Prospective studies are required to demonstrate the influence of normal carbohydrate metabolism on the frequency of infectious complications in this group.

\section{References}

1. Roberson JR, Raju S, Shelso J, Pui CH, Howard SC. Diabetic ketoacidosis during therapy for pediatric acute lymphoblastic leukemia. Pediatr Blood Cancer 2008; 50: 1207-12.

2. Sonabend RY, McKay SV, Okcu MF, Yan J, Haymond MW, Margolin JF. Hyperglycemia during induction therapy is associated with increased infectious complications in childhood acute lymphocytic leukemia. Pediatr Blood Cancer 2008; 51: 387-92.

3. Sonabend RY, McKay SV, Okcu MF, Yan J, Haymond MW, Margolin JF. Hyperglycemia during induction therapy is associated with poorer survival in children with acute lymphocytic leukemia. J Pediatr 2009; 155: 73-8.

4. Geerlings SE, Hoepelman AI. Immune dysfunction in patients with diabetes mellitus (DM). FEMS Immunol Med Microbiol 1999; 26: 259-65.

5. Janes SM, Barker KF, Mak V, Bell D. Invasive pulmonary aspergillosis in an insulin-dependent diabetic. Respir Med 1998; 92: 972-5.

6. Turina M, Fry DE, Polk HC Jr. Acute hyperglycemia and the innate immune system: clinical, cellular, and molecular aspects. Crit Care Med 2005; 33: 1624-33.

7. Tripathi BK, Srivastava AK. Diabetes mellitus: complications and therapeutics. Med Sci Monit 2006; 12: RA130-47.

8. Fuji S, Kim SW, Mori S, et al. Intensive glucose control after allogeneic hematopoietic stem cell transplantation: a retrospective matched-cohort study. Bone Marrow Transplant 2009; 44: 105-11.

9. Krinsley JS. Association between hyperglycemia and increased hospital mortality in a heterogeneous population of critically ill patients. Mayo Clin Proc 2003; 78: 1471-8.

10. Rassias AJ, Marrin CA, Arruda J, Whalen PK, Beach M, Yeager MP. Insulin infusion improves neutrophil function in diabetic cardiac surgery patients. Anesth Analg 1999; 88: 1011-6.

11. Van den Berghe G, Wouters P, Weekers F, et al. Intensive insulin therapy in the critically ill patients. N Engl J Med 2001; 345: 1359-67.

12. Van den Berghe G, Wouters PJ, Bouillon R, et al. Outcome benefit of intensive insulin therapy in the critically ill: Insulin dose versus glycemic control. Crit Care Med 2003; 31: 359-66.

13. Weiser MA, Cabanillas ME, Konopleva M, et al. Relation between the duration of remission and hyperglycemia during induction chemotherapy for acute lymphocytic leukemia with a hyperfractionated cyclophosphamide, vincristine, doxorubicin, and dexamethasone/ methotrexatecytarabine regimen. Cancer 2004; 100: 1179-85.

14. Koh GC, Peacock SJ, van der Poll T, Wiersinga WJ. The impact of diabetes on the pathogenesis of sepsis. Eur J Clin Microbiol Infect Dis 2012; 31: 379-88.

15. El Ansary MM, Allah NA, Emara NA, El Tablawy MM, Mahmoud S. Expression of (NM23-H1) gene in acute lymphocytic leukemia and its clinical significance. Arch Med Sci 2009; 5: 241-7.
16. Asturias EJ, Corral JE, Quezada J. Evaluation of six risk factors for the development of bacteremia in children with cancer and febrile neutropenia. Curr Oncol 2010; 17: 59-63.

17. Lowas SR, Marks D, Malempati S. Prevalence of transient hyperglycemia during induction chemotherapy for pediatric acute lymphoblastic leukemia. Pediatr Blood Cancer 2009; 52: 814-8. 